a

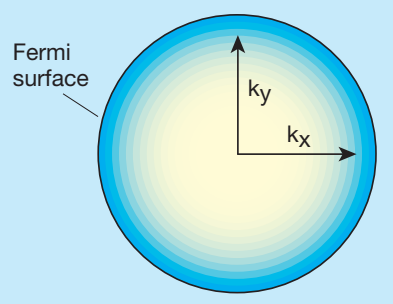

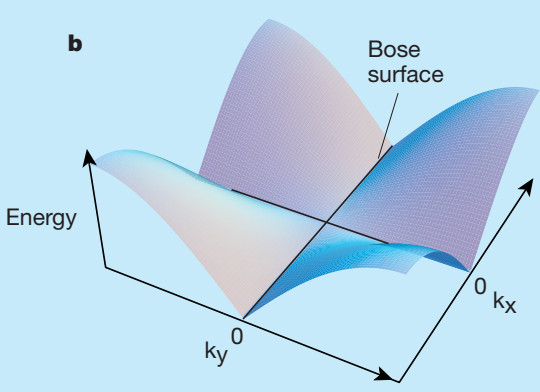

Figure 1 Fermi and Bose surfaces in two dimensions. a, Fermionic electrons in a metal obey Pauli's exclusion principle - there can be only one fermion in each quantum state. The Fermi surface marks the divide, defined by the particles' momentum components $\mathrm{k}_{\mathrm{x}}$ and $\mathrm{k}_{\mathrm{y}}$, between occupied and unoccupied momentum states. b, Although bosons do not obey the exclusion principle, Paramekanti et al. ${ }^{1}$ propose that they can form a Bose surface in a Bose liquid, analogous to the Fermi surface for fermions. In the spectrum of boson excitations in the Bose liquid, the excitation energy (vertical axis) vanishes near the Bose surface (which follows the lines $k_{x}=0$ and $k_{y}=0$ ): a Fermi liquid has a similar spectrum of excitations that vanishes on its circular Fermi surface.

To deter the bosons from forming a Bose-Einstein condensate or a Mott insulator, a more complex interaction between the bosons is necessary. In particular, a large contribution from 'boson ring exchanges' appears to be crucial. This feature arises because the quantum-mechanical description - or wavefunction - of the ground state contains a superposition of states in which pairs of bosons move in a correlated manner around a ring. In such a state, the positions of the bosons around the ring are uncertain, but a measurement that locates one boson at a particular site also specifies the position of the second boson at another site on the ring.

Stimulated by the proposal of Paramekanti et al., Sandvik et al. ${ }^{3}$ have already reported results from a computer simulation of the simplest quantum description of boson behaviour in two dimensions, including a large boson-ring-exchange term. So far, they have not found a state with a Bose surface, but have instead obtained a new form of Mott insulator in which the bosons of the lattice; this type of state had also been predicted ${ }^{4}$. crystallize on half the horizontal bonds
Paramekanti et al. ${ }^{1}$ also discuss the prospects for experimental discovery of their Bose liquid state. They suggest that such a state might be formed by pairs of electrons (which act like composite bosons and are known as Cooper pairs) in the cuprate compounds that superconduct at high temperatures. Mason and Kapitulnik ${ }^{5}$ have recently reported an unexpected regime of metallic conduction in a disordered thin film of $\mathrm{Mo}_{43} \mathrm{Ge}_{57}$ in a magnetic field - the film is a superconductor in zero field, and a Bose liquid of Cooper pairs is an intriguing possibility for the metallic phase. And there are other competing theories ${ }^{6-8}$, involving a more fundamental role for disorder in the film. It is clear that the resolution of the puzzle set by Paramekanti et al. brings the prospect of much exciting new physics.

Subir Sachdev is in the Department of Physics, Yale University, PO Box 208120, New Haven,

Connecticut 06520-8120, USA.

e-mail: subir.sachdev@yale.edu

1. Paramekanti, A., Balents, L. \& Fisher, M. P. A. Preprint cond-mat/0203171 (2002); http://xxx.lanl.gov. Phys. Rev. B (in the press)

2. Greiner, M., Mandel, O., Esslinger, T., Hansch, T. W. \& Bloch, I. Nature 415, 39-44 (2002)

3. Sandvik, A. W., Daul, S., Singh, R. R. P. \& Scalapino, D. J. Preprint cond-mat/0205270 (2002); http://xxx.lanl.gov

4. Park, K. \& Sachdev, S. Phys. Rev. B 65, 220405 (2002).

5. Mason, N. \& Kapitulnik, A. Phys. Rev. B 64, 060504

(2001).

6. Spivak, B., Zyuzin, A. \& Hruska, M. Phys. Rev. B 64, 132502 (2001).

7. Dalidovich, D. \& Phillips, P. Phys. Rev. Lett. 89, 027001 (2002).

8. Das, D. \& Doniach, S. Phys. Rev. B 64, 134511 (2001).

\title{
Physiology \\ Muscle regulator goes the distance
}

Vertebrate skeletal muscles — the kind that enable a mouse to run on a wheel or you to dash for a bus contain two types of fibre. Type I, or 'slow-twitch', fibres are fuelled mainly by oxidative metabolism, and can contract for sustained periods. Type II, 'fast-twitch' fibres generate rapid muscle contractions but are more prone to fatigue. On page 797 of this issue, Bruce Spiegelman and colleagues (Nature 418, 797-801; 2002) describe how a protein that regulates gene expression can increase the type I fibre content, and consequently improve the stamina of isolated mouse muscles.

Previous studies have shown that the nuclear protein PGC- $1 \alpha$ is involved in controlling genes that participate in energy metabolism in mammalian cells. This suggested that PGC- $1 \alpha$ might play a part in muscle physiology. Indeed, Spiegelman and colleagues have now found that if the protein is expressed in mouse skeletal muscles, more of the characteristically 'redder' type I muscle fibres are formed, with fewer type II fibres. The authors show that isolated PGC- $1 \alpha$ expressing muscles contain proteins that are typical of type I fibres, and can sustain contraction for around twice as long as equivalent muscles from wild-type mice, in an experiment based on standardized electrically induced contraction.

These results tie in nicely with the fact that the expression of PGC- $1 \alpha$ can be induced naturally by exercise in experimental animals, and that exercise can convert type II fibres into type I fibres. Further work

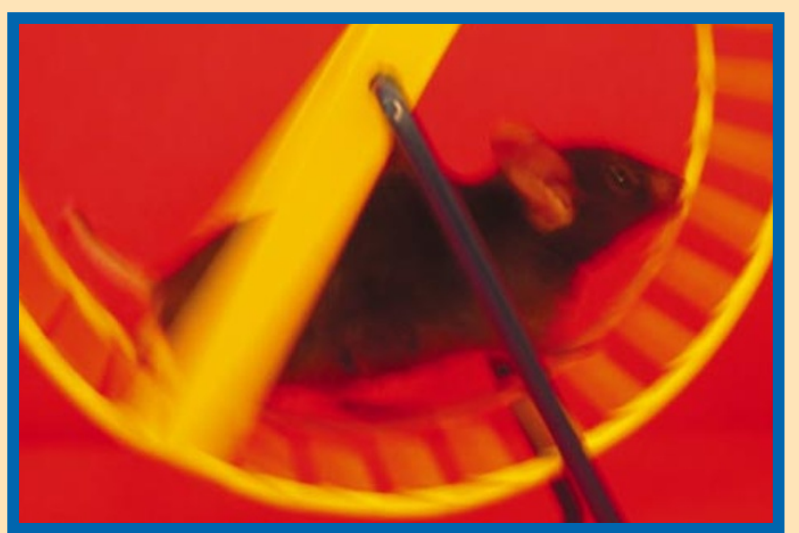

will be needed to define the precise molecular signals that control PGC- $1 \alpha$ activity and to identify the genes regulated by this protein that lead to the formation of type I muscle fibres. It may be that, in the future, drugs that influence these regulatory events will be useful in situations where muscle activity is impaired. Great care would have to be taken, however, as PGC- $1 \alpha$ seems to be involved in many other regulatory events in the body.
Richard Turner 